\title{
Expression of Immunoreactive Nitric Oxide Synthase Isoforms in Rat Kidney \\ Effects of Dietary Salt and Losartan
}

\author{
Akihiro Tojo, M.D., * Kirsten M. Madsen, M.D., Ph.D. \\ and Christopher S. Wilcox, M.D., Ph.D.**
}

\section{SUMMARY}

Immunohistochemical studies have shown expression of two different isoforms of NOS in the juxtaglomerular apparatus (JGA). Antibodies to a $\mathrm{Ca}^{++}$calmodulin dependent isoform purified from rat brain (B-NOS) label the macula densa cells whereas antibodies to an isoform purified from rat aortic smooth muscle cells in culture (VSM-NOS) induced with lipopolysaccharide and interferon $\gamma$ label the afferent arteriole. Since dietary salt intake and angiotensin II (Ang II) are determinants of renal NO generation, we have tested the hypothesis that salt intake can regulate the immunohistochemical expression of these NOS isoforms through an effect of Ang II. In 4 of 5 paired studies, the immunostaining for both B-NOS and VSM-NOS was more intense in rats that had received a low salt (LS), compared to a high salt (HS), diet. Infusion of the Ang II type $1\left(A T_{1}\right)$ receptor antagonist, losartan, enhanced the intensity of immunoreactive staining for both isoforms. In conclusion, the immunohistochemical expression of NOS isoforms in the JGA is increased by dietary salt restriction; this effect cannot be ascribed to Ang II acting on type 1 receptors. (Jpn Heart J 36: 389-398, 1995)

Key words: Nitric oxide synthase Juxtaglomerular apparatus Salt intake Angiotensin II Losartan

I MMUNOHISTOCHEMICAL localization of nitric oxide synthase (NOS) in the kidney was first demonstrated in the macula densa using antibodies directed against a constitutive, brain-type NOS isoform (B-NOS). ${ }^{1-3)}$ Antibodies to an inducible NOS isoform purified from rat vascular smooth muscle cells induced with lipopolysaccharide (LPS) and interferon $\gamma$ (INF- $\gamma$ ) (VSM-NOS) also

From Division of Nephrology, Hypertension and Transplantation, University of Florida, College of Medicine, Gainesville, FL and Department of Veterans Affairs Medical Center, 1601 SW Archer Road, Gainesville, FL, U.S.A.

Current address: *The 2nd Department of Internal Medicine, Faculty of Medicine, University of Tokyo, 73-1 Hongo, Bunkyo, Tokyo 113, Japan, **Division of Nephrology and Hypertension, Georgetown University Medical Centre, 3800 Reservoir Rd, NW-PHC F-6003, Washington D.C. 20007, U.S.A.

Address for Correspondence: Akihiro Tojo, M.D., The Second Department of Internal Medicine, Faculty of Medicine, University of Tokyo, 7-3-1 Hongo, Bunkyo-ku, Tokyo 113, Japan.

Received for publication January 27, 1995.

Accepted March 6, 1995. 
label the juxtaglomerular apparatus (JGA). However, immunoreactive VSMNOS was found in the terminal afferent arteriole and, to a lesser extent, in the initial efferent arteriole and the distal tubule. ${ }^{3)}$ Another isoform of inducible NOS, macrophage-type NOS, exists in the intercalated cells of the cortical collecting duct. ${ }^{4)}$ In support of those observations, mRNAs encoding two inducible isoforms of NOS have also been isolated from the tubules and arteries of normal rat kidney. ${ }^{5}$ )

Nitric oxide (NO) has several important functions in the kidney. These include the regulation of basal vascular resistance, ${ }^{6}$ the tubuloglomerular feedback (TGF) response, ${ }^{27}$ sodium excretion ${ }^{7)}$ and renin secretion. ${ }^{8)}$ Studies of the function of NO in the kidney have mostly relied upon the response to inhibition of NOS by substituted arginine analogs such as $\mathrm{N}^{\mathrm{G}}$-methyl-L-arginine (L-NMA). L-NMA infused into intact or isolated kidneys causes renal vasoconstriction with a reduction in the glomerular filtration rate (GFR) and a more pronounced reduction in the renal plasma flow (RPF). Using micropuncture methods, Deng and Baylis" found that intra-arterial L-NMA increased the preglomerular resistance and reduced the glomerular ultra-filtration coefficient (Kf). These studies have suggested that the afferent arteriole and the mesangium are major targets for $\mathrm{NO}$ action in the rat kidney. Ito et $\mathrm{a}^{10)}$ demonstrated directly that the afferent arteriole is constricted by L-NMA. However, this response is abolished by perfusing the macula densa with solutions that have a low $\mathrm{NaCl}$ concentration. These studies suggest that much of the action of $\mathrm{NO}$ on the afferent arteriole may derive from NO generated by macula densa cells activated by solute reabsorption. Indeed, we have found that microperfusion of L-NMA into the macula densa segment reduces the glomerular capillary hydraulic pressure (PGC), consistent with a predominant effect on the afferent arteriole. This response is blocked by co-perfusion with drugs that block macula densa cell transport. ${ }^{2}{ }^{2}$ This led to the hypothesis that macula densa-derived NO may mediate a vasodilator component to the TGF response. This function could be subserved by B-NOS in the macula densa, while VSM-NOS in the afferent arteriole and constitutive, endothelial cell NOS (EC-NOS) in the vascular endothelium could also contribute to the effects of $\mathrm{NO}$ on the regulation of afferent arteriolar resistance and/or renin release.

The sodium and chloride concentrations of the tubular fluid at the macula densa segment are the primary signals for activation of the TGF response. ${ }^{11,12)}$ Our recent studies have shown that a low dietary salt intake abolishes the vasodilator pathway mediated by NO between the macula densa and afferent arteriole. ${ }^{13)}$ Therefore, the first aim of these studies was to examine the hypothesis that the changes in dietary salt intake alters the immunoreactive expression of BNOS and VSM-NOS in the JGA. 
Whole-kidney studies have shown that blockade of Ang II generation with an ACE inhibitor or of Ang II type I (AT $)_{1}$ receptors with losartan can blunt or abolish the increase in renal vascular resistance produced by inhibition of NOS. ${ }^{14-16)}$ These results indicate that the renal vascular resistance is regulated in the balance between the vasodilators, such as $\mathrm{NO}$, and the vasoconstrictors, such as Ang II, and its increase by inhibition of NOS is mediated by Ang II. However, little is known about the direct effect of Ang II on NOS activity. Therefore, the second aim of these studies was to examine the hypotheses that Ang II acting on type I receptors also alters the immunohistochemical expression of B-NOS and/ or VSM-NOS in the kidney and that the changes in dietary salt intake alter NOS immunoreactivity via Ang II. To test these hypotheses we studied the effects of long-term changes in salt intake and infusion of an $A T_{1}$ receptor antagonist on the intensity of immunoreactivity for B-NOS and VSM-NOS in the juxtaglomerular apparatus.

\section{Methods}

Animal and tissue preparation: Male Sprague-Dawley rats weighing 250$300 \mathrm{~g}$ were maintained for 14 days on a regulated diet that differed only in the $\mathrm{NaCl}$ content (Teklad Inc., Madison, WI, USA). The high salt diet (HS) contained $6 \mathrm{~g} \cdot 100 \mathrm{~g}^{-1}$ of $\mathrm{NaCl}$ and the low salt (LS) diet contained $0.03 \mathrm{~g} \cdot 100 \mathrm{~g}^{-1}$ of $\mathrm{NaCl}$. This LS diet is sufficient for normal growth over a 2-3 week period.

All studies were paired. Thus, to assess the effects of dietary salt intake, pairs of LS and HS rats were prepared and studied identically. Five separate pair studies of HS and $\mathrm{LS}$ rats were undertaken. To study the role of $\mathrm{AT}_{1}$ receptors in mediating the effects of dietary salt, pairs of rats adapted to HS or LS diets were studied. One rat of each pair received a continuous subcutaneous infusion of losartan $\left(10 \mathrm{ng} \cdot \mathrm{kg}^{-1} \cdot \mathrm{min}^{-1}\right)$ from an osmotic minipump over seven days before perfusion-fixation while the other member of the pair received a vehicle infusion.

On the experimental day, rats were anesthetized with an intraperitoneal injection of pentobarbital $\left(50 \mathrm{mg} \cdot \mathrm{kg}^{-1}\right)$. The abdominal aorta was cannulated and the kidneys were perfused with phosphate buffered saline (PBS) to rinse out blood. They were preserved for immunohistochemical studies by perfusion with a paraformaldehyde-lysine-periodate (PLP) solution for $5 \mathrm{~min}$. The kidneys were cut into slices and immersed in the PLP solution overnight at $4^{\circ} \mathrm{C}$. After being rinsed with PBS, tissue was dehydrated in a graded series of ethanols and embedded in wax (Polyethylene glycol 400 disterate, Polysciences, Inc., Warrington, PA, USA).

Antibodies: A rabbit polyclonal antibody raised against a calcium-calmodulin dependent NOS isoform purified from rat brain was used to localize B-NOS in 
the rat kidney. The antibody was kindly provided by Dr. HHHW Schmidt, Northwestern University, Illinois, and has been extensively characterized previously. ${ }^{3,17-19)}$ A guinea pig polyclonal antibody raised against a NOS purified from rat aortic smooth muscle cells (VSM-NOS) pretreated for $12 \mathrm{~h}$ with a bacterial lipopolysaccharide (E. coli 0111: $30 \mu \mathrm{g} \cdot \mathrm{m} l^{-1}$ ) and rat recombinant interferon $\gamma$ (50 $\mathrm{ng} \cdot \mathrm{m} l^{-1}$ ) was used to localize inducible NOS. The NOS used for immunization of the guinea pig was purified to homogeneity as a single band on SDS/ PAGE. It was used as an immunogen in guinea pigs by direct lymph node administration. This antibody was kindly provided by Dr. SS Gross, Cornell University, New York, and its characterization has been described previously. ${ }^{3)}$ Immunohistochemistry: Wax sections $(4 \mu \mathrm{m})$ were processed for light microscopic immunohistochemistry using the avidin-biotin-horseradish peroxidase complex (ABC) technique (Vectastain ABC kit, Vector Laboratories, Burlingame, CA, USA) as described in detail previously. ${ }^{3)}$ Briefly, the sections were dewaxed, rehydrated and incubated for $30 \mathrm{~min}$ in $3 \% \mathrm{H}_{2} \mathrm{O}_{2}$ to eliminate endogenous peroxidase activity. After being rinsed in PBS, the sections were treated with blocking serum before incubation for $60 \mathrm{~min}$ with a primary antibody against BNOS (diluted 1:400) or VSM-NOS (diluted 1:500). Sections incubated without primary antibodies served as controls. The sections were rinsed with PBS and incubated with a biotinylated secondary antibody for $30 \mathrm{~min}$. After being rinsed, the sections were incubated for $30 \mathrm{~min}$ with Vectastain $\mathrm{ABC}$ reagent followed by incubation with the peroxidase substrate solution, diaminobenzidine and $\mathrm{H}_{2} \mathrm{O}_{2}$. The sections were counter-stained with hematoxylin. All the macula densa and the afferent arterioles in each section were observed, and the intensity of immunoreactivity shown in more than a half of JGA was considered as the representative immunoreactivity in each section, and photographed on a light microscope. The afferent arterioles were distinguished from the efferent arterioles by the width of arteriolar smooth muscle, and as we have reported previously, the antibody to VSM-NOS mainly labels the afferent arterioles. ${ }^{3)}$

\section{Results}

Effects of dietary NaCl intake on immunoreactivity for B-NOS and VSMNOS: In five separate studies, pairs of HS and LS rats were prepared and examined under identical conditions, except for the level of dietary salt intake. In four of the five pairs of studies, examination of immunostaining for B-NOS in the macula densa showed a more intense staining in the LS than the HS rats. Representative examples are shown in Figures 1A (LS) and 1B (HS). At both levels of dietary salt intake, immunostaining was prominent in the macula densa. Likewise, in four of the five paired studies, examination of the intensity of 


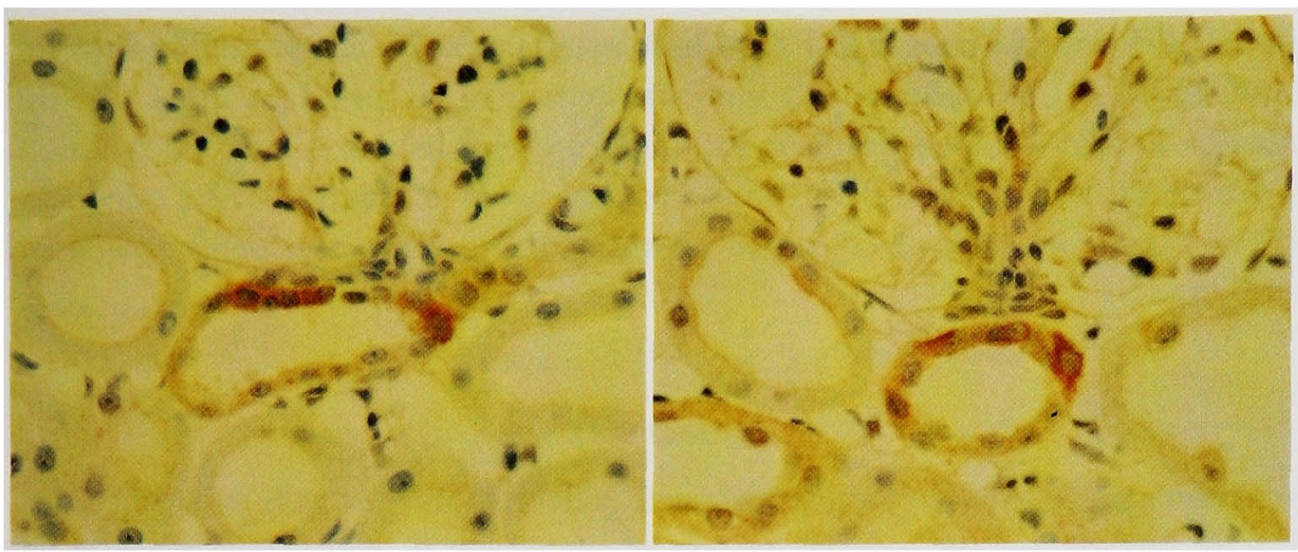

A

B

Figure 1. Light micrographs illustrating immunostaining for B-NOS in the macula densa of rats that were adapted to a low salt $(A)$ or a high salt $(B)$ diet $(\times 400)$. There is increased B-NOS immunoreactivity in animals on a low salt diet.

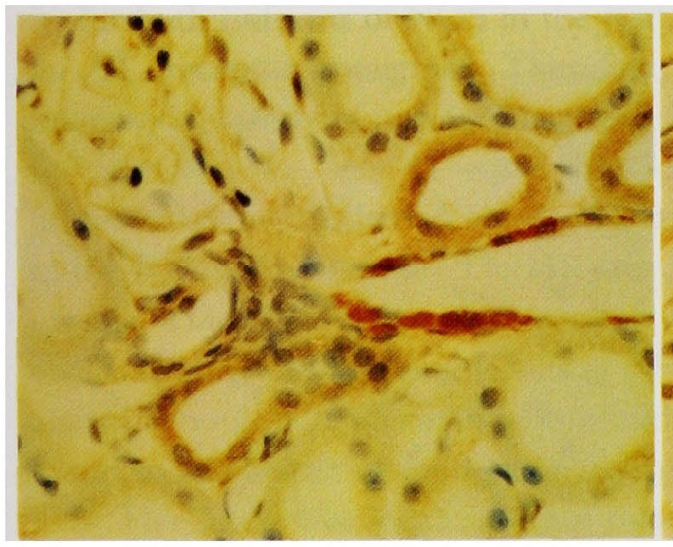

A

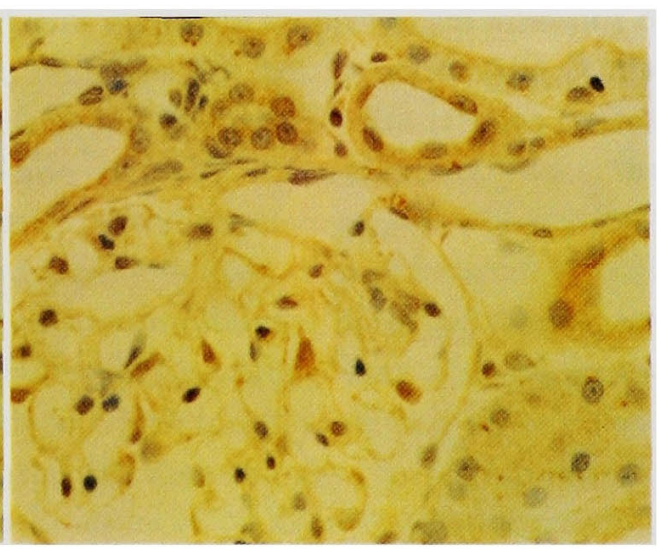

B

Figure 2. Light micrographs illustrating immunostaining for VSM-NOS in the terminal afferent arteriole of rats that were adapted to a low salt (A) or a high salt (B) diet $(\times 400)$. There is increased VSM-NOS immunoreactivity in animals on a low salt diet.

immunostaining for VSM-NOS in the terminal afferent arteriole showed clearly a more intense staining in the LS than the HS rat. Representative examples are shown in Figures 2A (LS) and 2B (HS). Immunostaining for VSM-NOS was most prominent in the afferent arteriole of all rats. However, some weaker and less consistent staining was also seen in the distal tubule cells. One pair of rats was discordant for both B-NOS and VSM-NOS immunoreactivity since, in this pair set, the immunostaining for both isoforms was rather more intense in the HS than the LS rat. 


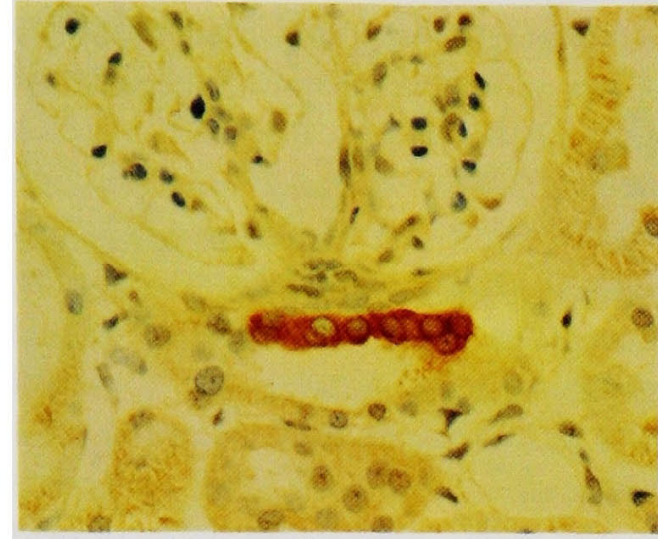

$\mathbf{A}$

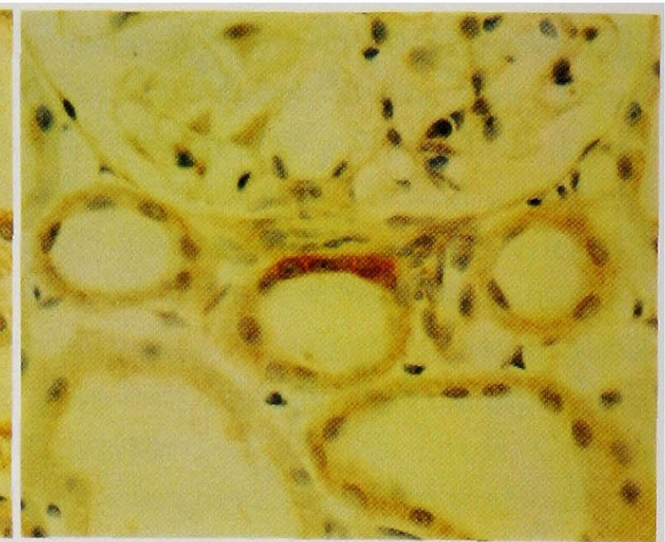

B

Figure 3. Light micrographs illustrating immunostaining for B-NOS in the macula densa of rats that were adapted to a low salt $(\mathrm{A})$ or a high salt $(\mathrm{B})$ diet. Both rats had received the $\mathrm{AT}_{\mid}$receptor antagonist, losartan, for 7 days prior to sacrifice $(\times 400)$. Treatment with losartan was associated with increased B-NOS immunoreactivity in both groups of animals (compare to Figure 1).

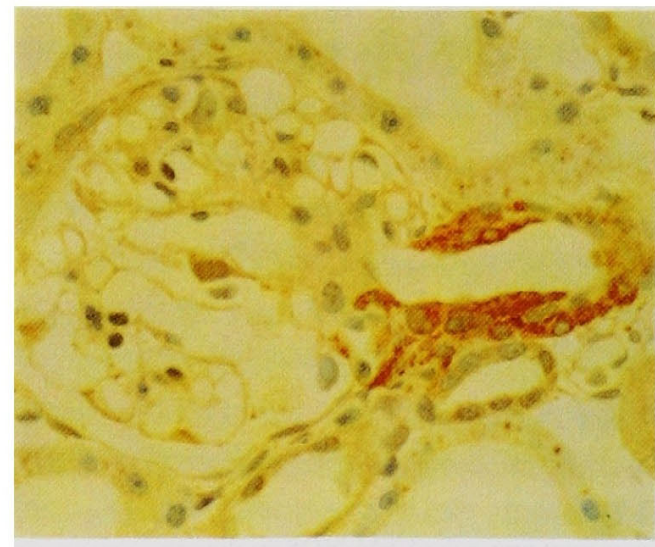

$\mathbf{A}$

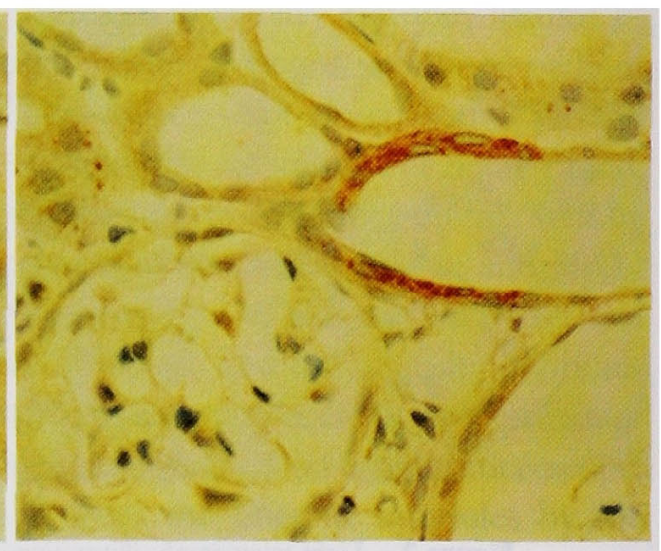

B

Figure 4. Light micrographs illustrating immunostaining for VSM-NOS in the terminal afferent arteriole of animals that were adapted to a low salt (A) or a high salt (B) diet. Both rats had received the $\mathrm{AT}_{1}$ receptor antagonist, losartan, for 7 days prior to sacrifice $(\times 400)$. Treatment with losartan was associated with increased VSM-NOS immunoreactivity in both groups of animals (compare to Figure 2).

Effects of losartan pretreatment: The effects of losartan pretreatment on immunostaining were studied in groups of LS and HS rats. There was a striking and consistent increase in the intensity of immunoreactive staining for both BNOS in the macula densa and VSM-NOS in the afferent arterioles. As shown in representative examples, the B-NOS staining was intense in the macula densa in both LS (Figure 3A) and HS (Figure 3B) rat kidneys. Likewise, VSM-NOS stain- 
ing was intense in the afferent arterioles of both LS (Figure 4A) and HS (Figure 4B) rat kidneys. Losartan increased not only the intensity of the VSM-NOS staining, but also extended the distribution of positively-staining cells proximally along the afferent arteriole. However, for both B-NOS and VSM-NOS immunoreactivity, there was still a greater staining in the JGA of LS compared to HS rats.

\section{Discussion}

These studies provide evidence for a distinct regulation of expression of NOS immunoreactivity in the kidney by dietary salt that appears independent of Ang II acting on $\mathrm{AT}_{1}$ receptors. In 4 of 5 studies of normal rats, the degree of immunoreactivity for both a constitutive NOS isoform in the macula densa and an inducible NOS isoform in the terminal afferent arteriole was stronger in those that had received a LS, compared to a HS, diet. The intensity of immunoreactivity for both isoforms of NOS in the JGA was increased by prolonged infusions of an $\mathrm{AT}_{1}$ receptor antagonist. Therefore, although LS diet increases Ang II, the increased intensity of immunoreactivity found in LS compared to HS rats cannot be ascribed to Ang II since blockade of $\mathrm{AT}_{1}$ receptors increases the immunostaining further. A greater intensity of immunostaining was still apparent in the JGA of LS compared to HS rats despite administration of losartan to both groups. Thus, the effects of dietary salt on immunostaining occurred despite any effects of Ang II. Opposing effects of dietary salt and Ang II may account for the aberrant result that was seen in one study in which immunostaining intensity was found to be greater in the kidney from the HS rat compared to the LS rat.

The distinctive localization of two isoforms of NOS in the JGA suggests a role for NO in the regulation of the TGF response. The concentration and rate of reabsorption of $\mathrm{NaCl}$ in the tubular fluid at the macula densa segment is the prime sensor that initiates the TGF response. ${ }^{11,12)}$ Expansion of the extracellular fluid volume (ECV) by an infusion of $\mathrm{NaCl}$ decreases the reabsorption of $\mathrm{NaCl}$ in the proximal tubule. However, despite an increased delivery of $\mathrm{NaCl}$ to the macula densa segment, the single nephron glomerular filtration rate (SNGFR) and the glomerular capillary hydraulic pressure $\left(\mathrm{PGC}_{\mathrm{GC}}\right)$ are increased, in part due to a blunting of the TGF response. Our preliminary studies have shown that blunting of the TGF response during a HS intake can be ascribed in part to an increased production of NO in the JGA that offsets vasoconstrictor signals generated by the TGF response. ${ }^{13)}$ Thus, the fall in PGC accompanying microperfusion of L-NMA into the macula densa segment was prevented in rats adapted to a LS diet. In the present morphologic studies, distal delivery of $\mathrm{NaCl}$ and TGF responses were not assessed. Therefore, a direct correlation between structure and 
function cannot be undertaken. Nevertheless, the results indicate that the absence of a functional response to perfusion of L-NMA into the JGA of LS rats seen in our preliminary studies cannot be ascribed to the disappearance of immunoreactive B-NOS or VSM-NOS from the macula densa or afferent arteriole. Indeed, the degree of immunostaining of these structures was increased in 4 of 5 pairs of studies in LS rats. Therefore, the absence of a functional response in these LS rats must be ascribed to some other factor not identified in these studies. Recently, we have found that microperfusion of L-arginine into the JGA causes a much greater increase in $\mathrm{PGC}$ in LS than HS rats. ${ }^{20)}$ Therefore, a decreased availability or metabolism of L-arginine by NOS, rather than a decreased expression of NOS immunoreactivity, may be the factor that limits NO generation in the JGA of LS rats.

Recently, it has been reported that small changes in ambient chloride concentration enhance nitric oxide release from cultured rat mesangial cells via constitutive NOS. ${ }^{21)}$ Even though these results demonstrate acute changes in nitric oxide production with reducing chloride concentration, it is possible that chronic restriction of chloride by feeding a low salt diet causes an increase in the production of nitric oxide with increased expression of NOS. This would be compatible with our demonstration of increased immunoreactivity of both constitutive B-NOS in the macula densa and inducible VSM-NOS in the terminal afferent arteriole of rats on a low salt diet, which could also increase the consumption of the substrate, L-arginine.

Studies of the effects of Ang II on the renal vascular response to inhibition of NOS have produced conflicting results. Thus, short-term blockade of Ang II with an ACE inhibitor or an $\mathrm{AT}_{1}$ antagonist has been reported to blunt, ${ }^{14-16)}$ or to leave unchanged, ${ }^{22-24)}$ the renal vasoconstrictor response to L-NAME or nitro-Larginine (L-NA). Short-term studies of the regulation of the renal microcirculation indicate that NO and Ang II may have counter-balancing actions on the afferent arteriole ${ }^{25)}$ However, during more prolonged perturbation of Ang II, rather different results have been obtained. Studies in two-kidney, one-clip $(2 \mathrm{~K}$, 1C) renovascular rats have shown that the renal vasoconstrictor response to inhibition of NOS is diminished in the post-clip kidney that has extremely high renin and Ang II levels. ${ }^{26)}$ The present studies were undertaken during relatively long-term changes in the ambient Ang II levels induced by dietary NaCl. The effects of losartan indicate that the immunoreactive expression of B-NOS and VSM-NOS in the JGA is diminished by Ang II acting on AT $_{1}$ receptors. Our results support the recent report which demonstrated that Ang II inhibits the cytokine-induced nitric oxide production and inducible NOS expression at both the protein and mRNA level via $\mathrm{AT}_{1}$ receptors in vascular smooth muscle cells ${ }^{27)}$ since we found that $\mathrm{AT}_{1}$ receptor antagonist markedly increased the expression 
of both B-NOS and VSM-NOS immunoreactivity in the JGA.

In conclusion, the results indicate that dietary salt regulates the immunoreactive expression of both B-NOS and VSM-NOS isoforms in the JGA via an effect that is independent of $\mathrm{AT}_{1}$ receptor activation. Since immunoreactive expression is only semiquantitative and does not correspond directly to NOS activity, further functional and biochemical studies are necessary to evaluate the significance of these findings.

\section{ACKNOWLEDGEMENTS}

This work was supported by a NIH grant (RO1-DK49870) and a merit review grant from the Department of Veterans Affairs. We are grateful to Harold H.H.W. Schmidt, Ph.D. for his generous gift of antibody to B-NOS and Steven Gross, Ph.D. for his generous gift of antibody to VSM-NOS.

\section{REFERENCES}

1. Mundel P, Bachmann S, Bader M, Fischer A, Kummer W, Mayer B, Kriz W: Expression of nitric oxide synthase in kidney macula densa cells. Kidney Int 42:1017, 1992

2. Wilcox CS, Welch WJ, Murad F, Gross SS, Taylor G, Levi R, Schmidt HHHW: Nitric oxide synthase in macula densa regulates glomerular capillary pressure. Proc Natl Acad Sci USA 89: 11993, 1992

3. Tojo A, Gross SS, Zhang L, Tisher CC, Schmidt HHHW, Wilcox CS, Madsen KM: Immunocytochemical localization of distinct isoforms of nitric oxide synthase in the juxtaglomerular apparatus of normal rat kidney. J Am Soc Nephrol 4: 1438, 1994

4. Tojo A, Guzman NJ, Garg LC, Tisher CC, Madsen KM: Nitric oxide inhibits bafilomycin-sensitive $\mathrm{H}^{+}$-ATPase activity in rat cortical collecting duct. Am J Physiol 267: F509, 1994

5. Mohaupt MG, Elzie JL, Ahn KY, Clapp WL, Wilcox CS, Kone BC: Differential expression and induction of mRNAs encoding two inducible nitric oxide synthases in rat kidney. Kidney Int 46: 653, 1994

6. Romero JC, Lahera V, Salom MG, Biondi ML: Role of endothelium-dependent relaxing factor nitric oxide on renal function. J Am Soc Nephrol 2: 1371, 1992

7. Mattson DL, Roman RJ, Cowley AW: Role of nitric oxide in renal papillary blood flow and sodium excretion. Hypertension 19: 766, 1992

8. Vidal MJ, Romero JC, Vanhoutte PM: Endothelium-derived relaxing factor inhibits renin release. Eur J Pharmacol 149: 401, 1988

9. Deng A, Baylis C: Locally produced EDRF controls preglomerular resistance and ultrafiltration coefficient. Am J Physiol 264: F212, 1993

10. Ito S, Johnson CS, Carretero OA: Modulation of angiotensin II-induced vasoconstriction by endothelium-derived relaxing factor in the isolated microperfused rabbit afferent arteriole. J Clin Invest 87: 1656, 1991

11. Lorenz JN, Weihprecht H, Schnermann J, Skøtt O, Briggs JP: Renin release from isolated juxtaglomerular apparatus depends on macula densa chloride transport. Am J Physiol 260: F486, 1991

12. Moore LG, lijima K, Rich A, Casellas D, Goligorsky MS: Communication of the tubuloglomerular feedback signal in the JGA. Kidney Int 39 (Suppl 32): S45, 1991

13. Welch WJ, Wilcox CS: Independent effects of salt intake and angiotensin II on the macula densa-nitric oxidc signalling pathway. J Am Soc Nephrol 4: 572, 1993

14. Sigmon DH, Beierwaltes WH: Angiotensin II: nitric oxide interaction and the distribution of blood flow. Am J Physiol 265: R1276, 1993 
15. Takenaka T, Mitchell KD, Navar LG: Contribution of angiotensin II to renal hemodynamic and excretory responses to nitric oxide synthesis inhibition in the rat. J Am Soc Nephrol 4:1046, 1993

16. Sigmon $\mathrm{DH}$, Carretero $\mathrm{OA}$, Beierwaltes $\mathrm{WH}$ : Angiotensin dependence of endothelium-mediated renal hemodynamics. Hypertension 20: 643, 1992

17. Schmidt HHHW, Pollock JS, Nakane M, Gorsky LD, Förstermann U, Murad F: Purification of a soluble isoform of guanylyl cyclase-activating-factor synthase. Proc Natl Acad Sci USA 88: 365, 1991

18. Schmidt HHHW, Warner TD, Ishii $\mathrm{K}$, Sheng $\mathrm{H}$, Murad $\mathrm{F}$; Insulin secretion from pancreatic $\mathrm{B}$ cells caused by L-arginine-derived nitrogen oxides. Science 255: 721, 1992

19. Schmidt HHHW, Gagne GD, Nakane M, Pollock JS, Miller MF, Murad F: Mapping of neural nitric oxide synthase in the rat suggests frequent colocalization with NADPH diaphorase but not with soluble guanylyl cyclase, and novel paraneural functions for nitrinergic signal transduction. $\mathrm{J}$ Histochem Cytochem 40: 1439, 1992

20. Welch WJ, Wilcox CS: Salt-sensitive regulation of $P_{G C}$ by macula densa L-arginine delivery. $J$ Am Soc Nephrol 5: 615, 1994

21. Tsukahara $\mathrm{H}$, Krivenko $\mathbf{Y}$, Moore LC, Goligorsky MS: Decrease in ambient [Cl] stimulates nitric oxide release from cultured rat mesangial cells. Am J Physiol 267: F190, 1994

22. Baylis C, Engels K, Samsell L, Harton P: Renal effects of acute endothelial-derived relaxing factor blockade are not mediated by angiotensin II. Am J Physiol 264: F74, 1993

23. Sigmon DH, Newman JM, Beierwaltes WH: Angiotensin II: endothelium-derived nitric oxide interaction in conscious rats. J Am Soc Nephrol 4: 1675, 1994

24. Deng $\mathrm{X}$, Welch WJ, Wilcox CS: Renal vasoconstriction during inhibition of NO synthase; effect of dietary salt. Kidney Int 46: 639, 1994

25. Ohishi K, Carmines PK, Inscho EW, Navar LG: EDRF-angiotensin II interactions in rat juxtamedullary afferent and efferent arterioles. Am J Physiol 263: F900, 1992

26. Sigmon DH, Beierwaltes WH: Renal nitric oxide and angiotensin II interaction in renovascular hypertension. Hypertension 22: 237, 1993

27. Nakayama I, Kawahara Y, Tsuda T, Okuda M, Yokoyama M: Angiotensin II inhibits cytokinestimulated inducible nitric oxide synthase expression in vascular smooth muscle cells. J Biol Chem 269: 11628,1994 